\title{
Comparing Effects of the Horizontal-Vertical Illusion on Grip Scaling and Judgment: Relative Versus Absolute, Not Perception Versus Action
}

\author{
Peter M. Vishton and Jacqueline G. Rea \\ Amherst College
}

\author{
James E. Cutting \\ Cornell University
}

\author{
Lisa N. Nuñez \\ Amherst College
}

\begin{abstract}
The discovery that the prehension component of an open-loop, two-fingered reach is largely immune to certain salient pictorial illusions has been used to suggest that humans possess 2 distinct visual systems, 1 that subserves perceptual judgment and 1 that mediates visually controlled action. In this article, the authors present evidence that suggests that the critical distinction is not that of reaching and judgment but of relative and absolute perception. Experiment 1 extends the findings of S. Aglioti, J. F. X. DeSouza, and M. A. Goodale (1995) and suggests that the manual prehension component of open-loop reaching is affected by the horizontal-vertical illusion to a much smaller degree than perceptual size judgments. In Experiments 2 and 3, however, when perceptual size judgment is directed at a single element of the display, this difference vanishes. Experiment 4 demonstrates that grip scaling is strongly affected by the illusion when a single reach is scaled to both the horizontal and vertical components of a triangular figure.
\end{abstract}

Inherent to the methodology of many studies of size and depth perception is the assumption that there exists a single, unified mental representation that is used for any and all types of size- and depth-mediated tasks. The hallmark of this type of study is the implicit assumption that the choice of behavioral measure is immaterial and that perception can be fully understood by simply asking experimental participants to verbally report their perceptions (for a variety of examples, see Goldstein, 1996). Many recent studies have revealed, however, that when the same perceptual issues are addressed by studies that use different task behaviors, very different results are often obtained, even if these task behaviors are based on precisely the same visual information (Brenner \& Smeets, 1996a, 1996b;

Peter M. Vishton, Jacqueline G. Rea, and Lisa N. Nuñez, Department of Psychology, Amherst College; James E. Cutting, Department of Psychology, Cornell University.

This work was supported in part by an Amherst College Faculty Research Grant and a National Research Service Award (MH1087105-1) from the National Institute of Mental Health. Revisions were completed with the support of the Institut des Sciences Cognitives, Centre National de la Recherche Scientifique, Lyon, France.

We thank Elizabeth Spelke, Barbara Finlay, and Daniel Huttenlocher for helpful discussions of and comments on earlier drafts of this article; David Field for the suggestion to examine the horizontal-vertical illusion; and James Todd for the suggestion to compare relative and absolute size perception. Comments and suggestions made on a previous version of this article by John Rieser were beneficial to the design of Experiment 4.

Correspondence concerning this article should be addressed to Peter M. Vishton, who is now at Tuck School of Business and Department of Psychology, Dartmouth College, HB 9000, Hanover, New Hampshire 03755-1014. Electronic mail may be sent to peter.vishton@ dartmouth.edu.
Bridgeman, Kirch, \& Sperling, 1981; Bridgeman, Lewis, Heit, \& Nagle, 1979; Castiello, Paulignan, \& Jeannerod, 1991; Goodale, Aglioti, \& DeSouza, 1994; Goodale, Milner, Jakobson, \& Carey, 1991; Loomis, Da Silva, Fujita, \& Fukusima, 1992; Post \& Welch, 1996; Proffitt, Bhalla, Gossweiler, \& Midgett, 1995; Smeets \& Brenner, 1995).

Aglioti, DeSouza, and Goodale (1995) reported a particularly striking example of how the choice of response behavior can greatly affect the results of a study. They compared the effects of the Titchener circles illusion (Figure 1) on two tasks: relative judgment and grip scaling. For the reasons described below, we focus in this article on the horizontal-vertical illusion (see Figure 2). First, however, a careful review of this previous work is essential.

When two target circles of equal size are surrounded by a circular array of either smaller or larger circles (Figures la and $\mathrm{lb}$ ), participants typically report that the target circle surrounded by the array of smaller circles appears larger than the one surrounded by the array of larger circles. This apparent difference in the two circles is referred to as the Titchener circles illusion. Participants in the Aglioti et al. (1995) study viewed pairs of Titchener circles displays presented on a horizontal surface with thin, plastic disks placed over the central circles (Figure 1). Trials in which the two discs were identical in size but appeared different (Figures la and lb) were randomly alternated with trials in which the sizes of the disks were made physically different such that they appeared to be identical in size (Figures lc and 1d). If, on viewing the display, participants judged that the circles were the same size, they were instructed to pick up the disk on the left; if their judgment was that the circles were different in size, they were instructed to pick up the disk on the right. 


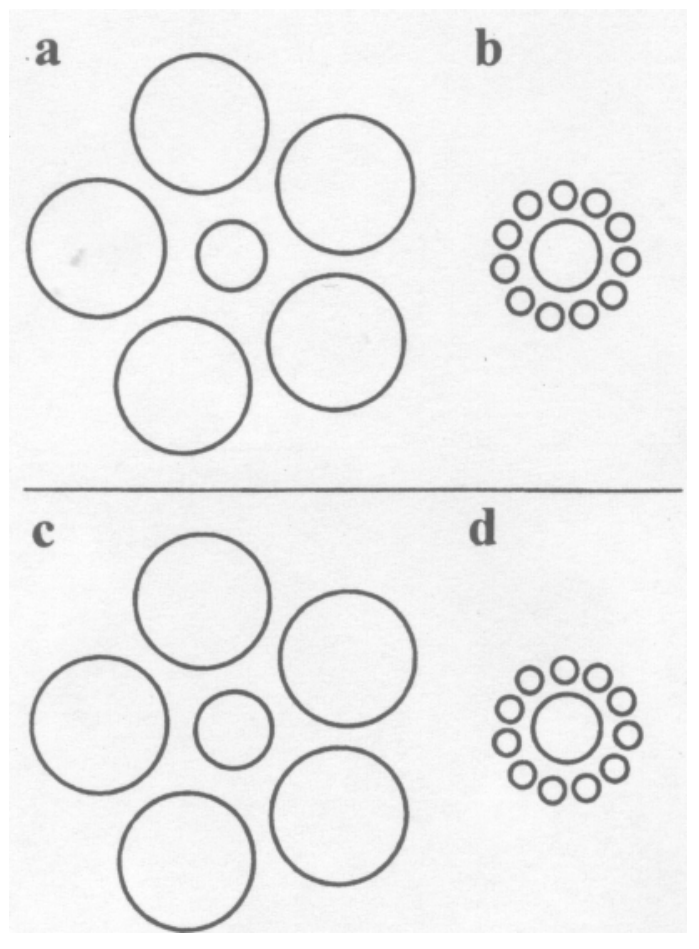

Figure 1. The Titchener circles illusion. Observers consistently judge the central circle in Figure $l b$ to be larger than the central circle in Figure la, even though the two central circles are, in fact, identical in size. Most observers would judge the central circles in Figures lc and 1d to be nearly identical, even though Figure lc is $10 \%$ larger in diameter (Aglioti et al., 1995).

The choice of whether to reach left or right, on the basis of this relative judgment, was thus recorded as a measure of participants' conscious perceptions of the displays. Infrared light-emitting diodes were attached to the index finger, thumb, and wrist of the participant to measure the dynamics of the reaches themselves. As described in other research (e.g., Castiello \& Jeannerod, 1991; Castiello et al. 1991; Gentilucci, Daprati, Gangitano, Saetti, \& Toni, 1996; Gentilucci, Daprati, Toni, Chieffi, \& Saetti, 1995; Servos, Goodale, \& Jakobson, 1992), the configuration of the fingers during a reach is precisely coordinated with the size of the target. By recording the distance between a participant's thumb and index finger as a two-fingered reach for the central disk was made, a second measure of the participant's perception of the target size was obtained. Whereas the choice task revealed a clear and large effect of the illusion on conscious perception, the distance between the fingers was nearly unaffected by it. A variety of experimental results such as this make it clear that any complete characterization of human visual information processing should describe not only the properties of the stimulus but the properties of the response behavior as well.

Aglioti et al. (1995) related this result to a body of neurological findings that suggest that there are two distinct visual streams that accomplish two distinct types of processes: (a) a dorsal stream of projections from primary visual cortex (and superior colliculus via the pulvinar) to the posterior parietal cortex, which mediates visually controlled action, and (b) a ventral stream from primary visual cortex to the temporal lobe, which subserves conscious perception (Goodale \& Milner, 1992; Goodale et al., 1991; see also Ungerleider \& Mishkin, 1982).

Aglioti et al. (1995) attributed their result to a difference in the operating characteristics of these two systems. They claimed that the dorsal stream relies on a restricted set of visual information (perhaps binocular cues), excluding whatever source or sources of information give rise to the Titchener circles illusion. Conversely, the ventral stream is described as making use of a broad class of information, including that which causes the illusion to occur: "This result suggests that the automatic and metrically accurate calibrations required for skilled actions [italics added] may depend on visual computations that are different from those driving our perceptual judgments [italics added] about objects" (Aglioti et al., 1995, p. 684).

Such statements imply that the key difference between the two tasks is that one involves an overt perceptual judgment, whereas the other involves a motor action. This is a thoroughly plausible explanation, but it is certainly not the only one. On comparing the reaching and relative judgment conditions used by Aglioti et al. (1995), a variety of other confounding differences between the two tasks can be identified. Five are highlighted below.

1. The experimental task involved reaching for an object that provided tactile feedback to the participants at the conclusion of each reach. This would not influence the participants' reaches on the very first trial of the experiment, but it may have supported learning over the course of the study. The reaching system may have thus been adapted to scale its size perceptions on the basis of this proprioceptive feedback. If this were verified, it would not diminish the importance of the findings. It would, in fact, indicate that the reaching system does use information that the conscious judgment system does not use. In order to properly compare the size perception used for judgment with visually con-
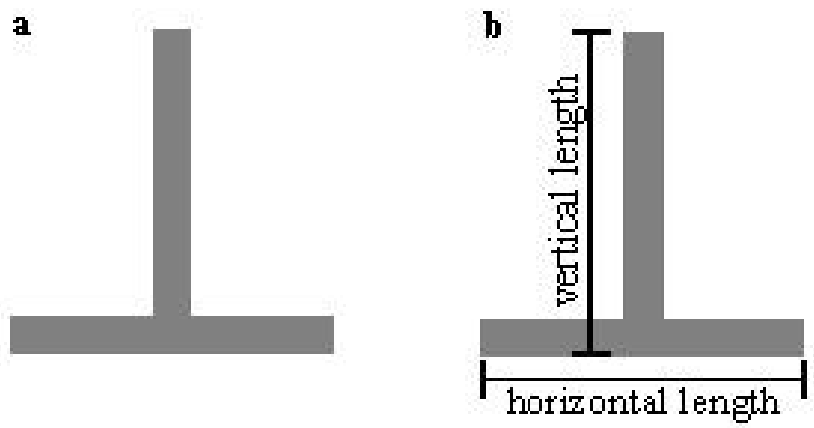

Figure 2. The horizontal-vertical illusion. (a) The horizontal and vertical lines are identical in length when measured as implied here, but most observers report that the vertical line appears to be considerably longer (Avery \& Day, 1969). (b) Before all procedures, it was made clear to participants that for any and all tasks, the extent of the vertical line length was assumed to continue to the very bottom of the figure. 
trolled grip scaling, however, no informative feedback should be given during the testing portion of the study.

2. The participants did not perform a truly open-loop reaching task because the hand was in view during at least the final portion of the reach. There is some evidence that rapid, ballistic reaching actions are preplanned before the movement commences and do not make use of on-line visual information (i.e., in a closed-loop fashion) even if it is provided (e.g., Jakobson \& Goodale, 1991; Jeannerod, 1984, 1988). This same research indicates that this inherently open-loop character is specifically true for the scaling of the maximum grip aperture observed near the midpoint of the reaching motion. Because this was the primary measure used by Aglioti et al. (1995), they felt that participants were not using the appearance of the hand to calibrate the grip. It could be, however, that some effect of the illusion is exerted during the final phase of the reach. It is also very possible that the appearance of the hand may provide some feedback that influences the performance of subsequent trials.

3. To effectively reach an object, a hand must move to a target without bumping into any obstacles along the way. In the Titchener circles illusion, the target stimuli are surrounded by additional elements that may influence the planning of the movement without affecting the perception of the target object size (e.g., Howard \& Tipper, 1997; Tipper, Howard, \& Jackson, 1997). Thus, a circle seen as very large may induce a very closely aimed grip if it is surrounded by closely spaced external barriers (Figure lb), whereas a reach aimed for a circle seen as small may not induce a tightly aimed grip if there are large spaces into which the fingers may be placed (Figure la). Taken as a whole, the summation of [large size + close grip] may very well equal [small size + relaxed grip] and result in the effects observed by Aglioti et al. (1995). Again, if this were the case, the studies would not be devalued. Evidence that the reaching system is affected by two-dimensional lines as if they were barriers would be an interesting finding. Nonetheless, the possibility that size perception is not the only important facet of this task has not been considered.

4. The reaching target in the Aglioti et al. (1995) study is three-dimensional, whereas the illusion-inducing elements are two-dimensional. The immunity to the illusion may result from the reaching system separating the threedimensional stimuli from the two-dimensional illusioninducing elements. Ideally, an experimental stimulus would contain target and illusion-inducing elements that are of the same dimensionality.

5. The study measured maximum grip aperture, whereas the ultimate goal of grip scaling is the aperture at the end of the reach. Previous studies have demonstrated a strong correlation between the maximum grip aperture and the final grip aperture (e.g., Jakobson \& Goodale, 1991; Jeannerod, 1984, 1988); however, it is possible that the Titchener circles illusion may exert its effects on the final stages of the reach. Ideally, an experiment would examine the ultimate goal of a reach-its ending position.

By presenting reaching participants with a truly openloop task, without tactile or visual feedback, without crowding elements surrounding the target, and with two-dimensional illusion-inducing elements, and then measuring the ending state of participants' grip scaling, we have explored in Experiment 1 whether any of these five problems could have caused the striking distinction reported by Aglioti et al. (1995).

\section{Experiment 1}

In this study, participants performed open-loop reaching and judgment tasks with the inverted-T displays associated with the horizontal-vertical illusion (Figure 2; Avery \& Day, 1969). When horizontal and vertical lines of equal length are arranged in this configuration, most observers report that the vertical appears to be longer than the horizontal. In order for the two lines to be perceptually "equal" in length, the horizontal must typically be $120 \%$ as long as the vertical (Avery \& Day, 1969).

\section{Method}

Participants. Eight volunteer participants ( 4 men and 4 women) from the Cornell University community were recruited through posted sign-up sheets. Eleven participants (4 men and 7 women) recruited from the Amherst College community completed an alternate version of the initial reaching procedure. Eight additional participants ( 4 men and 4 women), recruited from the Amherst College community, completed a final control study. All participants had normal or corrected-to-normal visual acuity.

Stimuli and apparatus. Displays were presented on a flat, horizontal table surface. Although most studies of the horizontalvertical illusion have presented stimuli in the frontoparallel plane, we used this horizontal orientation to allow for a closer comparison with the reaching task and the experiments of Aglioti et al. (1995).

Participants sat in a chair facing the table throughout the study. Inverted-T displays associated with the horizontal-vertical illusion (Figure 2) were printed in black ink (Personal Laser Writer 320, Apple Computer, Cupertino, CA) on sheets of standard white paper (DP $20 \mathrm{lb} .4200$, Xerox, Webster, NY), which were cut to $15.0 \times 21.6$ $\mathrm{cm}$. All lines were $1.25 \mathrm{~mm}$ in width. Circle targets used in the control study were printed in the same way. All displays were placed on the table immediately in front of the observer, approximately 50 $\mathrm{cm}$ from the participant's eyes.

Prior to beginning the reaching task, small metal nubs (approximately $0.5 \mathrm{~mm}$ thick and $1.5 \mathrm{~mm}$ tall) were attached, using adhesive tape, to the index finger and thumb of the participant's preferred hand. Before each trial, these metal nubs were pushed through a paper card into an ink pad, causing the metal tips to become coated with ink while the rest of the finger remained dry. The nubs were carefully adjusted such that the participants would leave two small dots (approximately $0.1 \mathrm{~mm}$ in diameter) as their fingers touched a piece of paper at the end of a reach.

Design. In the primary procedure, participants conducted judgment and open-loop reaching tasks with nine different displays: (a) one equal-line-length display, in which both the horizontal and vertical extents were $41 \mathrm{~mm}$, (b) four vertical variations, for which the horizontal extent was $41 \mathrm{~mm}$ and the verticals were $25,33,49$, and 57 $\mathrm{mm}$, and (c) four horizontal variations, for which the vertical extent was $41 \mathrm{~mm}$ and the horizontals were 12, 27, 53, and 63, $\mathrm{mm}$. This range of line lengths was chosen such that, for both the shortest vertical variation and the widest horizontal variation, the horizontal line would clearly be perceived to be longer than the vertical line. 
With each of these nine displays, participants made two reaches for the vertical line and two reaches for the horizontal line. Participants additionally made two size judgments about the vertical line and two size judgments about the horizontal line for each display. Four of the participants began with the reaching task; 4 participants completed the judgment task first. Within each task, the display order was randomized by a thorough shuffling of the printed pages.

For the secondary procedure, three displays were produced in which the horizontal and vertical extents were an equal 31,51 , or $71 \mathrm{~mm}$. Participants made two reaches for each horizontal and two reaches for each vertical. Displays were randomly ordered using the same shuffling procedure.

For the reaching control study, participants made four reaches each for 31-, 51-, and 71-mm circles. For two of these reaches, participants reached with a horizontally oriented grip; for two reaches, the grip was vertical.

Procedure. At the beginning of the reaching task, the metal nubs were affixed to the fingers, and the participant practiced making dots on a blank piece of paper. Prior to each reaching trial, the participant would apply ink to the markers and then move his or her hand out of view, below the table surface. At this point, the experimenter would place the next stimulus sheet on the table in front of the participant and instruct him or her to reach out and touch the ends of a particular line, as if reaching to pick up a very thin object. Participants were explicitly instructed to make certain that their eyes were closed before beginning to move their hand and to keep them closed until the hand had been retracted and placed out of sight below the table. The experimenter sat facing the participant to make sure that this was done. If the eyes opened at any point while the hand was in view, the trial was discarded and the participant was reinstructed to not look at the stimulus at any time during the reach. This occurred on less than $1 \%$ of experimental trials. As the participant retracted his or her hand, the experimenter removed the display sheet from the table such that the participant could not see the dots he or she had made. A clean stimulus sheet was used for each trial. The distance between the two small dots made on each piece of paper was later measured by hand to the nearest millimeter and recorded for analysis.

For the judgment task, participants were seated in the same position used for the reaching task with the hands again held out of view. For the horizontal judgment trials, participants were instructed to complete the following sentence: "If the vertical line is 100 units in length, the horizontal line is _ units long." For the vertical judgment trials, participants were instructed to complete the converse sentence: "If the horizontal line is 100 units in length, the vertical line is _ units long."

The entire primary procedure lasted for approximately $30 \mathrm{~min}$. The secondary and reaching control study procedures, in which only reaching data were collected, required approximately $15 \mathrm{~min}$ each.

\section{Results}

The length judgment task provided a clear replication of the horizontal-vertical illusion effect (Avery \& Day, 1969). Figure 3 depicts participants' mean judgments of the vertical/horizontal length ratios as a function of the true vertical/horizontal ratio. The mean slope of this function was significantly greater than $1: M=1.17 ; S E=0.12 ; t(7)=$ $26.75, p<.001$. The intercept of this line for the vertical/horizontal ratio of 1 (the ratio perceived for a display in which the horizontal and vertical lines are equal) was also significantly greater than $1: M=1.20 ; S E=0.03$; $t(7)=18.98, p<.001$.

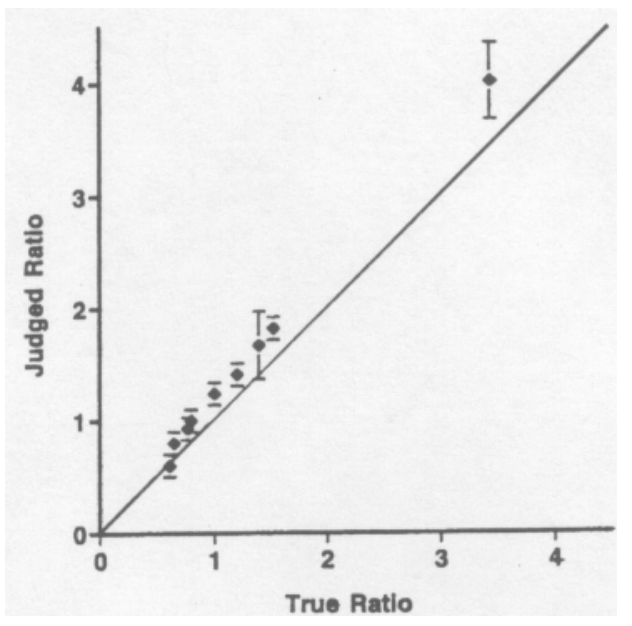

Figure 3. Mean vertical-horizontal ratio judgments for Experiment 1. The judgment task of Experiment 1 provided a clear replication of the horizontal-vertical illusion effect. When the vertical-horizontal ratio of the line length was 1.0, participants judged this ratio to be approximately 1.2 . The gray line indicates the region of veridical performance; vertical error bars indicate \pm 1 SEM.

The grip-scaling component of the reaching task was typically accurate, but the placement of the hand was not. The dots left by the fingers were an average of $64 \mathrm{~mm}$ from the nearest line end. The true mean is likely to have been much greater than this, however, because the most inaccurate $8 \%$ of the trials were lost when participants missed the stimulus paper entirely. It has previously been noted that reaching movements typically include an initial ballistic component, followed by a corrective motion near the end of the reach (e.g., Goodale, Pelisson, \& Prablanc, 1986). Because the participants' eyes were closed, the relatively low accuracy of the hand placement may be explained by the lack of visual information necessary to make this correction.

In contrast to this, with regard to the grip-scaling component of the reach, Goodale, Jakobson, and Keillor (1994) have reported that $2 \mathrm{~s}$ of visual disruption are required to affect accuracy. Because the participants in this study made their reaches immediately after closing their eyes, requiring far less than $2 \mathrm{~s}$, it is therefore not surprising that the scaling of the grip remained quite accurate. Our remaining analyses focus on this size-mediated component of the action.

For each participant, a least squared error regression line was fit to the data relating finger gap size to target size. The slope of this line for the horizontal stimuli was slightly greater than that for the vertical (horizontal: $M=1.21, S E=$ 0.25 ; vertical: $M=1.20, S E=0.09$ ), but this effect was far from significant, $t(7)=0.05, p=.96$. Figure 4 depicts the mean ratio of the vertical and horizontal finger gaps produced for each display as a function of the true ratio. For each participant, a least squared error regression line was fit to these data. The mean slope was less than $1(M=0.85, S E$ $=0.19$ ), and the intercept of this line with 
the true horizontal-vertical ratio of 1 was slightly less than $1(M=0.99, S E=0.13)$. However, neither of these differences approached significance (slope: $p=.46$; intercept: $p$ $=.93$ ).

This pattern of results suggests that, whereas judgment is strongly, consistently affected by this illusion, grip scaling is not. However, because of a high level of variance in the reaching data, particularly for 2 of the 8 participants, the interaction across tasks was not significant: slope-- $F(1,7)$ $=2.54, p=.16$; intercept with ratio of $1--F(1,7)=2.81, p$ $=.14$. To allow for a more concrete assessment of this trend, an additional group of participants was recruited to participate in the secondary reaching procedure. By more precisely affixing the metal marking tips, by encouraging the participants to more carefully practice making dots on a blank page prior to the study, and by concentrating the data collection on a smaller number of displays, a large reduction in this variance was obtained.

The results of the secondary procedure were somewhat different from those of the primary procedure. The first procedure suggested a small effect (which did not approach significance) that ran counter to the horizontal-vertical illusion. Contrary to this, the results of the secondary procedure indicated a small but significant increase in the finger gaps of reaches for the vertical line as compared with the horizontal. The mean slope of the regression line relating finger gap size to the vertical line was slightly greater than that for the horizontal: vertical $M=1.02, S E=$ 0.10 ; horizontal $-M=0.86, S E=0.13, t(11)=1.57, p=.14$ (see Figure 5). The mean ratio of the vertical and horizontal finger gaps was significantly greater than $1: M=1.03 ; S E$ $=0.12 ; t(11)=2.33, p=.04$. When the ratios produced by the reaching of this second, more carefully assessed group

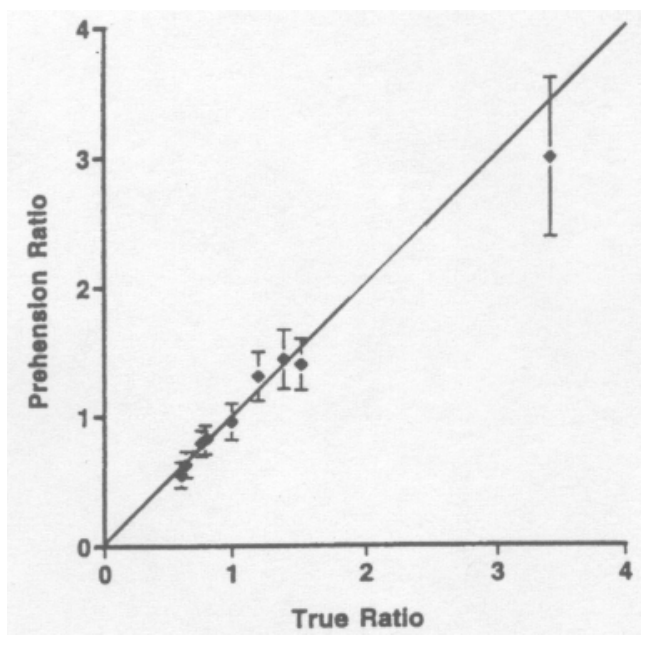

Figure 4. Mean vertical-horizontal finger gap ratio for Experiment 1. Data from the primary reaching procedure indicate no effect of the horizontal-vertical illusion. The finger gap ratios produced in the secondary reaching procedure, however, reveal a significant effect of the horizontal-vertical illusion on grip scaling. This effect is significantly smaller than that observed for perceptual judgment. The gray line indicates the region of veridical performance; vertical error bars indicate \pm 1 SEM.

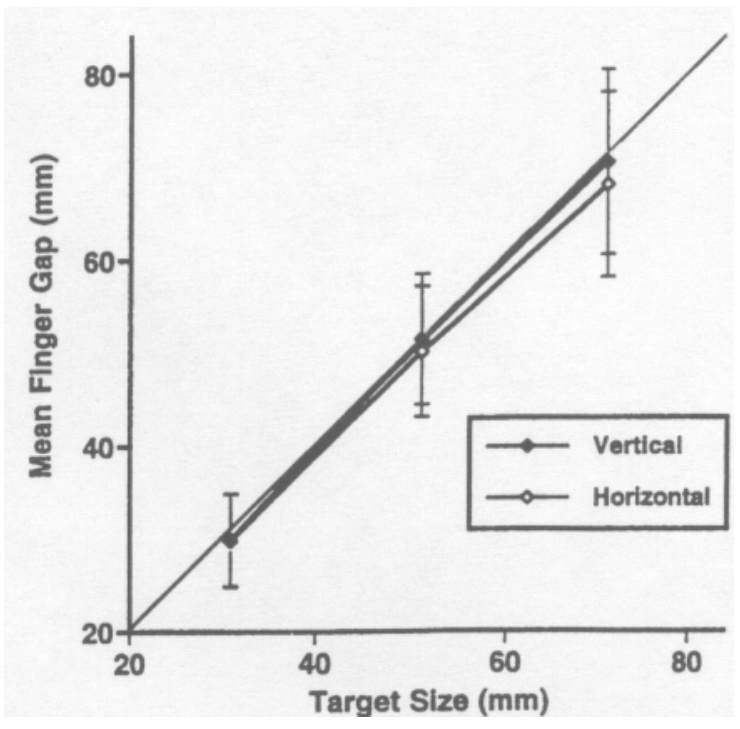

Figure 5. Mean finger gaps for the secondary reaching procedure. Finger gaps associated with reaches for the vertical line were slightly larger than the gaps for horizontal reaches. The gray line indicates the region of veridical performance; vertical error bars indicate \pm 1 SEM.

are compared with judgments made about the original equal ratio stimuli, a clear difference in the magnitude of the horizontal-vertical illusion emerges, $t(18)=12.73$, $\mathrm{p}<$ .001 .

The reaching control study provided an assessment of the effect of the grip orientation on finger gap size. No clear effect emerged. The mean ratio of vertical and horizontal finger gaps was .99: $S E=0.10, t(7)=0.10, p=$ .92 .

\section{Discussion}

The judgment task data indicate that when the horizontal and vertical lines of equal length are presented under these experimental conditions, participants perceive the vertical line to be approximately $120 \%$ as long as the horizontal. Conversely, if the horizontal line is $120 \%$ as long as the vertical, participants perceive the lines as approximately equal in length. For comparison with other measures and other experiments, we refer to this as a $20 \%$ illusory effect.

The secondary procedure suggests that grip-scaling performance is also affected by this illusion, but to a much lesser extent. When the horizontal and vertical lines are equal in length, the reaches made for the vertical line result in finger gaps that are an average of $103 \%$ as wide as those accompanying reaches for the horizontal. Conversely, if the horizontal line is $103 \%$ as long as the vertical, then the finger gaps associated with reaches will be approximately equal. The horizontal-vertical illusion can thus be said to have a $3 \%$ effect on grip scaling.

When reaching for a vertically oriented target, the wrist must be rotated to a much different degree than when reaching for a horizontal target, a factor that provides a potential explanation for the reduction of the illusory effect on the grip-scaling task. Perhaps the wrist rotation associ- 
ated with vertical reaches causes the finger gap to close slightly; perhaps the wrist rotation associated with horizontal reaches causes the finger gap to slightly increase; perhaps both. To test for this, the reaching control study was run in which participants aimed vertically and horizontally oriented reaches at circles. No orientation effects were apparent.

Castiello, Bonfilioli, and Bennett (1996) have reported differences in prehensile reaching actions when participants are directed at some two- and three-dimensional stimuli (e.g., three-dimensional apple and a two-dimensional image of that apple). To our knowledge, however, no study has suggested that reaches directed at thin plastic disks $(3 \mathrm{~mm}$ thickness), such as those used by Aglioti et al. (1995), should be markedly different than reaches aimed at two-dimensional stimuli. Of all of the participants who were instructed to reach for the patterns printed on the paper as if to pick up a very thin object, not a single person voiced a concern that he or she could not reach for a two-dimensional stimulus. Although it is possible that our data were affected by the use of two-dimensional stimuli, the accurate, consistent grip scaling suggests that this task is appropriate for assessing the effects reported by Aglioti et al. Similarly, if closing their eyes affected the participants' ability to maintain an accurate representation of the target size or to use that information to mediate the reaching action, then much greater inaccuracies would have been apparent.

This study puts to rest all five of the concerns raised about the methods used by Aglioti et al. (1995). When participants were presented with a truly open-loop task, without tactile or visual feedback, without crowding elements surrounding the target, and with two-dimensional illusion-inducing elements, their grip-scaling performance indicates a clear reduction in the effect of this pictorial illusion.

Findings such as this have typically been interpreted as evidence of a distinction between two visual pathways (e.g., Goodale \& Milner, 1992): one that mediates the grip-scaling task (the action system) and a second that mediates the judgment task (the perception system). An inference strongly implied by this idea is that any grip-scaling task will be only slightly affected by the Titchener circles or horizontal-vertical illusion, whereas any judgment task will be greatly affected.

This perception versus action theory provides a plausible explanation for these findings, but any of several remaining differences between the judgment and reaching tasks may be primarily responsible. The most apparent distinction is that the grip-scaling task is directed at only one stimulus element, whereas the judgment task is directed at two. In the study reported by Aglioti et al. (1995), this experiment, and nearly all other previous studies of the horizontal-vertical or Titchener circles illusions, observers have judged the relative size of two or more stimulus elements (e.g., Avery \& Day, 1969; Cormack \& Cormack, 1974; Glaser \& Slotnick, 1995; Higashiyama, 1992, 1996; Kubi \& Slotnick, 1993; Teghtsoonian, 1972; but see Verrillo \& Irvin, 1979; Yang, Wade, \& Proffitt, 1998). In the experiment reported by Aglioti et al., participants judged which of two stimuli was larger. In Experiment 1 of the present article, the judgment of the size of the vertical line was made in terms of the horizontal length and vice versa. In the reaching task, however, participants were instructed to direct their attention and responses to a single element of the display and to indicate their perception of size in absolute terms. ${ }^{1}$

Many studies have suggested distinctions between these two types of perception (Cutting, 1986; Todd \& Norman, 1995; Todd \& Reichel, 1989), which may result in the differential illusory effects. To directly compare the effects of the horizontal-vertical illusion on judgment with its effects on reaching, a task is needed in which participants' responses are similarly mediated by the absolute size of a single display element.

\section{Experiment 2}

Participants began this procedure by learning to accurately judge the lengths of horizontal or vertical lines in millimeters. After judging the length of each line, participants were informed of the line's true length and of the magnitude and sign of their error. These errors were initially large, but over the course of a few trials, participants became quite accurate. All of this feedback occurred prior to the presentation of any illusion-inducing stimulus. A variety of different explanations have been offered for why the horizontal-vertical display makes the vertical line appear approximately $20 \%$ longer than its identical horizontal counterpart; all explanations involve either (a) the presence of the horizontal line making the vertical line appear longer or (b) the presence of the vertical line making the horizontal line appear shorter. ${ }^{2}$ If this approach is correct, then the addition of the second line to the stimulus should have altered participants' perceptions of the length of the first line and consequently impacted their judgments of its metric size. By comparing the direction and magnitude of this effect to the grip-scaling effect of Experiment 1, it was possible to assess the extent to which the horizontal-vertical illusion affects grip scaling and conscious judgment differently. By comparing the direction

\footnotetext{
${ }^{1}$ The term absolute is potentially confusing in this context because any size judgment must be made in reference to something else (in this case, standard metric units). For the purposes of this article, the term relative describes a judgment or grip scaling that is mediated by the ratio of two sizes. The term absolute indicates that the grip scaling or judgment is made in reference to the size of one, and only one, element of a display. Although participants' responses were presumably scaled to an internal notion of how large a millimeter is or how large one's own hand is, we have chosen to use this terminology because both task behaviors are mediated by the absolute size of a single display element.

${ }^{2}$ Several studies have shown effects of orientation on the perceived length of single lines that were consistent with the horizontal-vertical illusion (e.g., Schiffman \& Thompson, 1975; Verrillo \& Irvin, 1979). This seems to be part of the cause of the illusion, but these effects are not large enough to fully account for the illusion. Explanations offered for why the horizontal-vertical illusion is larger than the values obtained in these experiments have always considered the effects that one line has upon the perceived size of the other. One exception to this has recently been reported by Yang et al. (1998) for larger stimuli viewed from a distance of 45 feet. The current studies consider only smaller viewing distances (within one arm length).
} 
and magnitude of this effect to the judgment effect of Experiment 1, it was possible to assess the extent to which the horizontal-vertical illusion affects relative and metric judgment differently.

\section{Method}

Participants. Ten participants (6 women and 4 men) were recruited from the Amherst College community and received course credit for participation. All participants had normal or corrected-to-normal visual acuity.

Stimuli and apparatus. Displays were presented and data were collected with an Indy Workstation (Model 84600, Silicon Graphics, Mountain View, CA). Lines were displayed in white on a black background and were again $1.25 \mathrm{~mm}$ wide. Judgments of line length were made in millimeter units and entered using the keyboard. During training, after each response was entered, participants were told the true line length and the direction and magnitude of their error in judgment. To give the participant a sense of his or her improvement, a graph depicting the progression of errors made over the course of the study was then presented for $3 \mathrm{~s}$.

Design and procedure. All participants completed separate training and testing sessions for vertical and for horizontal line stimuli. Half completed the vertical task first; half began with the horizontal task. During each of 50 training trials, a single line appeared on the computer screen, the length of which was chosen randomly from the whole number values between 10 and $109 \mathrm{~mm}$. Participants estimated the length of this line in millimeters, entered that value, and received feedback, after which the next trial commenced. Each training procedure required approximately $15 \mathrm{~min}$ and was followed immediately by the corresponding testing procedure. At the start of these trials, participants were told that no more feedback would be given and that a second line would appear on the screen during certain trials. It was emphasized, however, that their task was exactly the same as it had been in the training procedure-to judge the length of a single line.

Test trial line lengths were set at each of the intervals of four between 10 and $110 \mathrm{~mm}$ inclusive (i.e., 10, 14, 18, . . 106, 110). Each line length was presented twice: once as a single line and once with the perpendicular line added. The testing procedure required approximately $15 \mathrm{~min}$, during which time 52 trials (26 lengths x 2 illusion conditions) were completed in a random order. After completing the first training-testing procedure, participants took a brief rest and then continued with the second procedure.

\section{Results}

Figure 6 depicts the estimates of size made for the horizontal and vertical lines during the training procedure. Some learning seemed to take place, but a high level of accuracy was achieved very quickly. The initial 10 horizontal size estimates exhibited a mean error magnitude of $6.55 \mathrm{~mm}(S E=0.40)$, which was an average of $13 \%$ of the line length $(S E=0.91)$. The initial 10 vertical errors had a mean magnitude of $7.22 \mathrm{~mm}(S E=0.41)$, which was an average of $18 \%$ of the line length $(S E=0.95)$. In the middle 40 trials, do clear improvement was apparent as the mean error was $13 \%(S E=1.00)$ for the horizontal judgment task and $15 \%(S E=0.72)$ for the vertical. Even in the final 10 trial, the mean errors were only slightly decreased: $12 \%$ ( $S E$ $=0.97)$ and $15 \%(S E=0.66)$ for the horizontal and

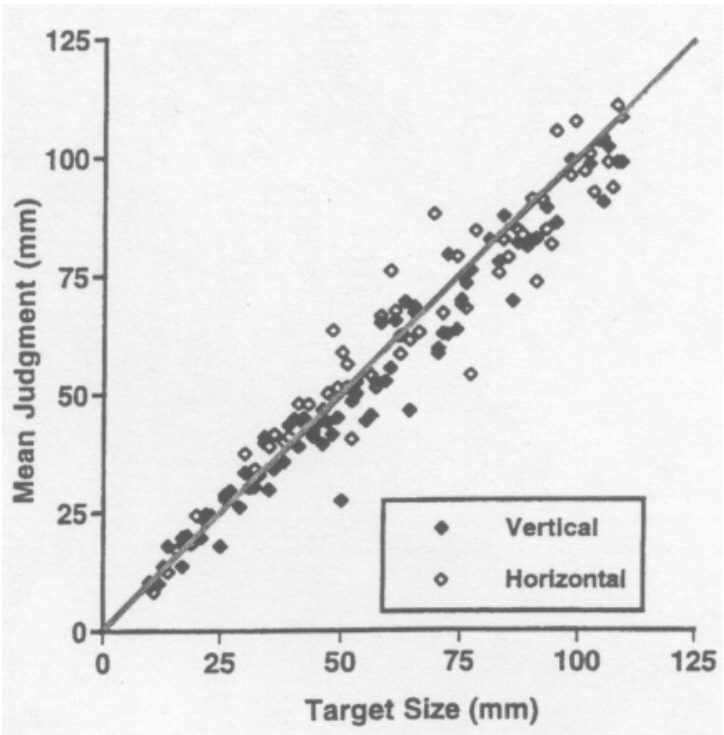

Figure 6. Mean size judgments made for vertical and horizontal line lengths during the training procedures in Experiment 2. The gray line indicates the region of veridical performance.

vertical, respectively. In this final phase of the training procedure, $95 \%$ of the participants' horizontal judgment errors fell within $14 \%$ of the actual line length; $95 \%$ of the vertical errors were less than $16 \%$. Over the course of the testing procedure, in which no feedback was given, judgment accuracy remained very consistent. The mean slope of the regression line relating participants' error magnitude to trial number for test trials was positive for only 4 of the 10 participants $(M=0.02, S E=0.03)$.

The left panel of Figure 7 depicts the mean horizontal size judgments made for different line lengths during the testing phase, grouped by the illusion condition; the right panel of Figure 7 shows the same for the vertical judgment task. The mean judgment for the single horizontal line condition was $102 \%$ ( $S E=2.38)$ of the true length of the line. The mean judgment for the horizontal-vertical condition was $105 \%$ ( $S E=1.87)$. Thus, participants typically overestimated the size of the horizontal line in both conditions, slightly more in the illusion condition. Neither of these values was significantly different from $100 \%$, and they were not significantly different from each other, but the trend runs counter to the horizontal-vertical illusion. Confidence interval calculation indicates that we can be 95\% certain that the vertical line decreases the perceived absolute size of the horizontal line by less than $1 \% .^{3}$

The mean judgments for the vertical in the single line and the illusion conditions were $102 \%(S E=1.28)$ and $104 \%(S E=0.88)$. Thus, consistent with the horizontal-vertical illusion, the overestimation of the vertical line size was slightly increased by the presence of the horizontal. This effect was not significant, but we can

\footnotetext{
${ }^{3}$ This and all subsequent confidence intervals were calculated using two-tailed Student's $t$ values for the appropriate degrees of freedom.
} 

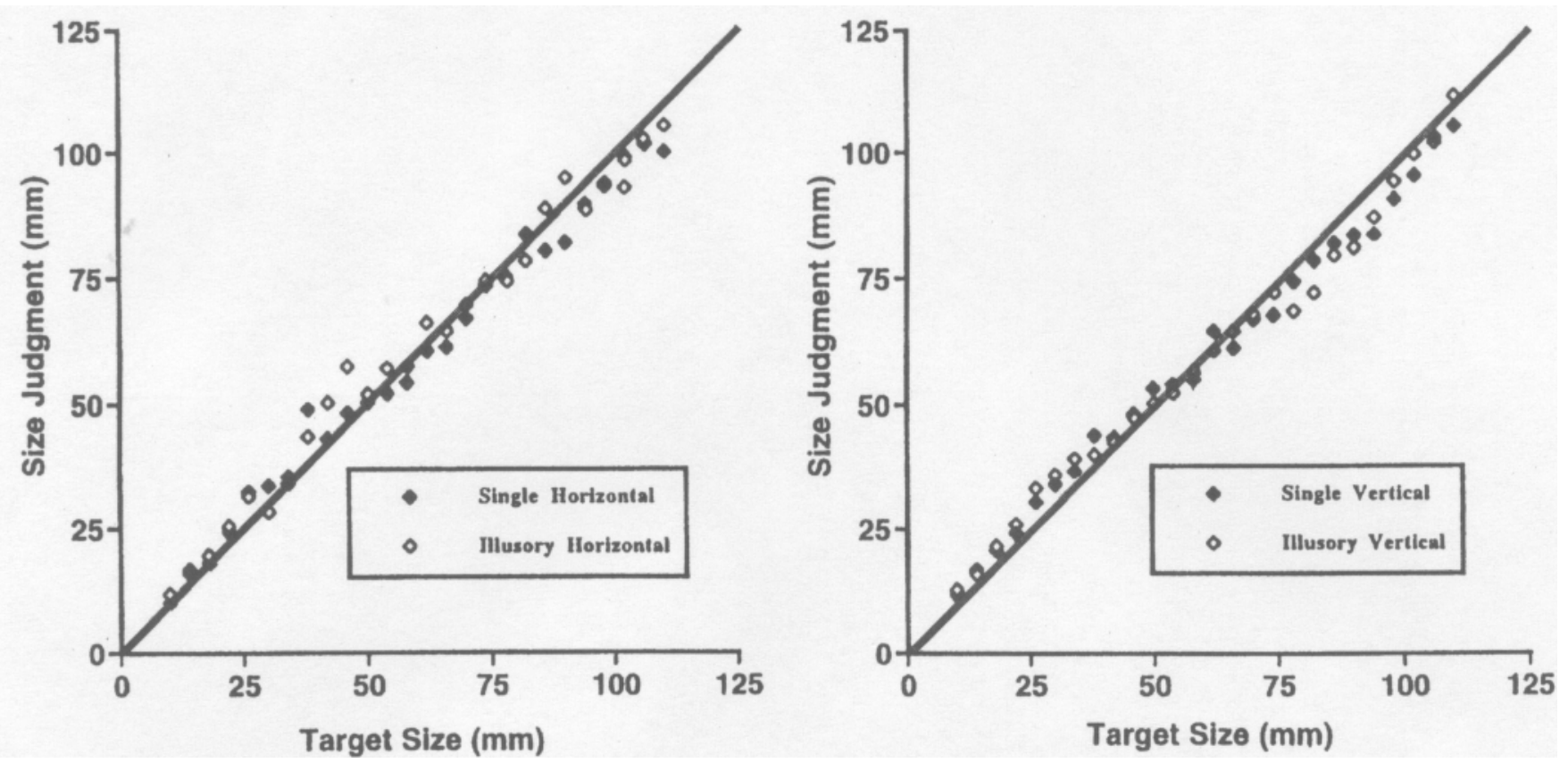

Figure 7. Mean size judgments of horizontal (left panel) and vertical (right panel) line lengths in single line and illusion conditions. The gray line indicates the region of veridical performance.

be $95 \%$ certain that the true increase was less than $3 \%$.

\section{Discussion}

In all four of the test conditions, participants tended to overestimate the size of the lines, but not significantly so. The data collected here do not allow for a confident statement that the horizontal-vertical illusion has any effect on metric size judgment, but they do allow us to confidently state that the size of any effect is small. If the presence of the vertical line causes the horizontal line to appear no more than $1 \%$ shorter than its true size and if the presence of the horizontal line causes the vertical line to appear no more than $3 \%$ longer than its vertical size, then the overall effect of the horizontal-vertical illusion on metric size perception is less than $4 \%$. Even the bounds of the $99 \%$ confidence intervals indicate that the effect is less than 7\%. It seems that conscious perceptual judgment can be just as immune to the horizontal-vertical illusion as grip scaling if the judgment task is also mediated by the absolute size of a single element of the display.

\section{Experiment 3}

One potential cause of the reduction of the illusory effect in Experiment 2 is the feedback provided during the training trials. Although it should be emphasized that all of the training occurred prior to the presentation of the illusion inducing stimuli, perhaps this feedback resulted in a more robust, illusion-resistant size perception process. Participants' judgments would probably be more variable in the absence of feedback, but the same is probably true for grip scaling. In every trial of the Aglioti et al. (1995) studies, participants received tactile feedback about the absolute size of the three-dimensional reaching target. Outside the lab, our participants receive feedback of this sort every time they make a visually directed reach. Perhaps the distinction between grip scaling and judgment performance is not one of perception versus action but one of well-practiced versus unpracticed behaviors.

To assess this hypothesis, we had participants in Experiment 3 make conscious size judgments that were based on a single element of a display, using a straightforward, nonlinguistic task for which they did not receive any feedback. After viewing a stimulus, participants marked off a segment of a separate line such that it appeared to be the same length as the stimulus line.

\section{Method}

Participants. Forty-eight volunteers (20 women and 28 men) were recruited from the Amherst College community and offered a candy bar in return for their participation. All participants had normal or corrected-to-normal visual acuity.

Stimuli and apparatus. Displays were presented on a flat, horizontal table surface. Participants sat in a chair facing the table throughout the study. The inverted-T displays were printed on individual sheets of paper and were identical to those used in Experiment 1 with the following exceptions. Each inverted-T display was equal in height and width; stimulus line lengths of 15, 30, 45, 60, and $75 \mathrm{~mm}$ were used. Size judgments were marked on sheets on which 10 long response lines $(186 \times 1.25 \mathrm{~mm})$ were printed. For half of the participants, these response lines were oriented horizontally; for the other half, the paper was rotated such that the lines were vertical. Test displays were presented approximately $70 \mathrm{~cm}$ from the viewer's eyes. Response line sheets were 
placed on the table immediately in front of the observer, approximately $50 \mathrm{~cm}$ from the participant's eyes.

Design and procedure. The papers containing the stimuli were thoroughly shuffled for each participant. Participants in the horizontal response condition were told that they should indicate each size perception by making a small mark on the response line such that the distance between the left end of the line and the mark was the same as the length of the indicated stimulus line. Participants in the vertical response condition similarly placed horizontal marks an appropriate distance from the top end of the response line. It was emphasized that judgments should be based on, and only on, the indicated stimulus line. After each mark was made, an opaque cover sheet was placed to prevent participants from looking back at previous responses.

Twenty-one participants made judgments about the horizontal lines; 27 participants judged vertical line length. Of the 21 participants in the horizontal stimulus line condition, 8 indicated their judgments on horizontal response lines, and 13 indicated their judgments on vertical response lines. Of the 27 participants in the vertical stimulus line condition, 13 indicated their judgments on horizontal response lines, and 14 indicated their judgments on vertical response lines. Each participant made two separate judgments of each of the five lengths for a total of 10 trials.

\section{Results}

No significant effects of response line orientation were noted; data were therefore collapsed across these two conditions for the following analyses. Figure 8 depicts the estimates of size made for the horizontal and vertical lines of the inverted-T displays.

For the horizontal stimulus lines, marked segments averaged $99 \%$ ( $S E=0.02$ ) of line length; for the verticals, the mean was $102 \%(S E=0.02)$. For the 15-, 30-, 45-, 60-,

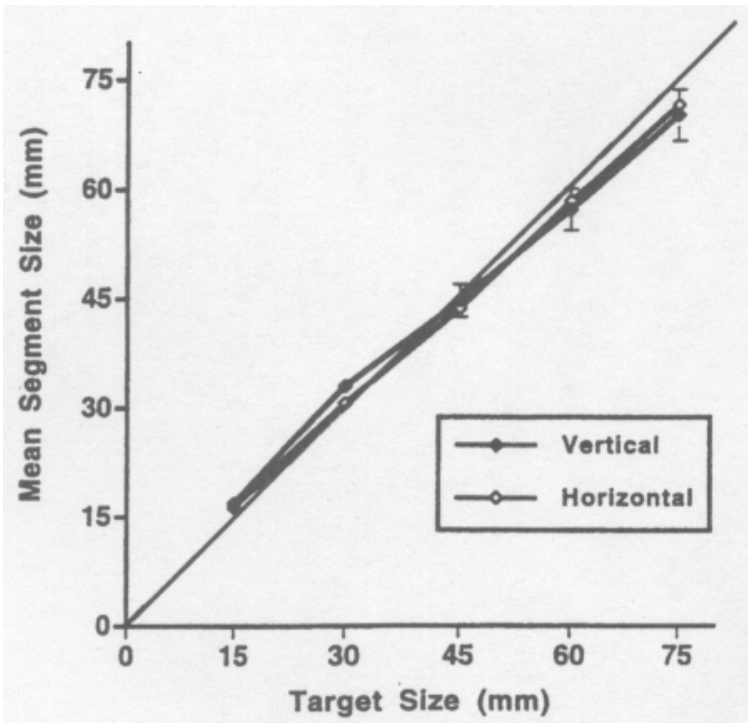

Figure 8. Mean segment size. In the line segmentation task, participants overestimated the length of small stimuli and underestimated the length of large stimuli. As with all absolute judgment tasks reported in this article, however, the effects of the horizontal-vertical illusion were very small. The gray line indicates the region of veridical performance; vertical error bars indicate \pm 1 SEM. and $75-\mathrm{mm}$ vertical stimuli, the means were $113 \%(S E=$ $3.9), 110 \%(S E=2.3), 99 \%(S E=2.1), 95 \%(S E=1.5)$, and $94 \%(S E=2.0)$. For the horizontal stimuli, the means were $109 \%(S E=3.9), 102 \%(S E=3.3), 97 \%(S E=2.7)$, $97 \%(S E=2.4)$, and $91 \%(S E=2.7)$. For small stimuli, observers tended to overestimate the length of the stimulus lines; for large stimuli, underestimation occurred. Repeated measures analysis of variance (ANOVA) indicated a significant effect of size, $F(4,184)=27.0, p<.001$. Neither the effect of the illusion, $F(1,46)=1.06, p=.31$, nor the interaction between stimulus size and illusion, $F(4$, $184)=1.49, p=.21$, were significant.

What is primarily relevant to the hypothesis at hand is the ratio of the vertical judgments to the horizontal judgments. The ratio of mean horizontal to mean vertical estimates was a very small 1.03--a 3\% effect of the illusion. Recall from Experiment 1 that relative judgments of such stimuli indicated a $20 \%$ difference in perceived length. Even if we are very conservative in our estimates of these means, a dramatic reduction in illusory effect is apparent. On the basis of the data collected, we can be $95 \%$ certain that the true mean vertical estimate was below $106 \%$ of line length and that the true mean horizontal estimate was greater than $96 \%$ of line length. We can therefore be $95 \%$ certain that the ratio of these judgments is less than 105.5/96.1 $=1.097$, a $10 \%$ illusory effect.

\section{Discussion}

As in the previous two experiments, mean differences in this line-segmenting task suggest an effect of the horizontalvertical illusion. The effect, however, was substantially less than that observed for relative judgment and only slightly greater than the effect of the illusion on grip scaling. The increase in the size of the effect between Experiments 2 and 3 suggests that the feedback stage of Experiment 2 did affect the data, but it was clearly not necessary in order for the horizontal-vertical illusion to be greatly reduced in magnitude.

In Experiments 1 to 3, whenever size judgments were directed at a single element of a display, the horizontalvertical illusion exerted only a small effect. This was equally true for reaching and for judgment behaviors. Directly implied by this emphasis on the distinction between relative and absolute perceptual judgments is the prediction that if a reaching task is mediated by two or more elements of a display, then the horizontal-vertical illusion should exert a large effect on grip scaling, just as it does when judgment is mediated by multiple elements of a display.

\section{Experiment 4}

Most studies of grip scaling have focused on two-fingered reaching actions. This is not an uncommon action, and many studies have shown that it is precisely scaled to the size of a target stimulus. Many reaching actions, however, involve all five fingers. As humans reach out to grasp a target object, all five fingers are shaped into a configuration 
that approximates the size and shape of the target (Jeannerod, 1994, 1997; Jeannerod, Arbib, Rizzolatti, \& Sakata, 1995).

An object shaped like the outline depicted in Figure 9a could be picked up in many ways. One method that is commonly volunteered by naive participants is depicted in Figure 9c. To quickly and effectively pick up such an object, the distance between the index and middle fingers must be scaled to the width of the horizontal line; the distance between the fingers and the thumb must similarly be scaled to the vertical extent of the object. If one reaches for the square stimulus depicted in Figure $9 \mathrm{~b}$ using a similar action, the three-fingered reach must also be influenced by both the height and width of the object.

In the key condition of this experiment, participants viewed two-dimensional images of the shapes depicted in Figures $9 \mathrm{a}$ and $9 \mathrm{~b}$ and then made open-loop, closed-eye reaches according to the procedure used in Experiment 1. Participants attempted to reach as if to pick up a very thin object, placing the fingers as suggested by Figures 9c and $9 \mathrm{~d}$, with the thumb at the lower corner of the triangle or midpoint of the square and two fingers on the upper corners of the figure.

\section{Method}

Participants. Twenty-three volunteers (13 women and 10 men) from the Amherst College community received course credit for
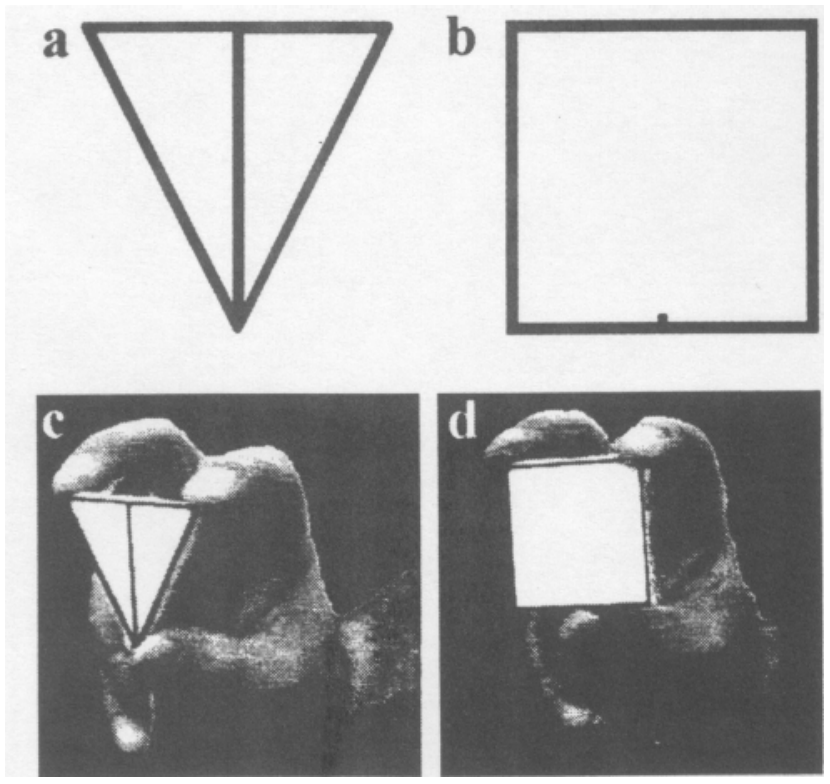

Figure 9. Figures 9a and 9b depict the two-dimensional stimuli used in this study. Participants viewed the printed stimuli and reached for them as if to pick up a thin object by using the grip depicted in Figures 9c and 9d. Effectively performing this action requires that the distance between the two fingers be scaled to the stimulus width, whereas the distance between the fingers and thumb must be scaled to the stimulus height. The finger configuration thus contains a simultaneous indicator of the visually registered size of the horizontal and vertical extent of the figures. their participation. All participants had normal or corrected-to-normal visual acuity.

Stimuli and apparatus. Dark gray triangles and squares (Figures 9a and $9 \mathrm{~b}$ ) were printed with equal heights and widths of 31 and $37 \mathrm{~mm}$ (Personal Laser Writer 320, Apple Computer, Cupertino, CA) on sheets of standard white paper (DP $20 \mathrm{lb}$. 4200, Xerox, Webster, NY). All lines were $1.5 \mathrm{~mm}$ in width. Post and Welch (1996) have presented data indicating that when reaching actions are aimed at directly visible targets, they do not function in the same way as when directed at indirectly specified targets (e.g., a corner as opposed to the middle of a line). The small mark placed at the center of the base of the square (Figure 9b) provided a directly visible target for the thumb placement, so as to make the reaching actions more comparable. Test displays were placed on a table directly in front of the participant, approximately $50 \mathrm{~cm}$ from the viewer's eyes. To record the grip scaling of two-fingered and three-fingered reaches, participants wore a thin, latex glove (Microtouch, Johnson \& Johnson, Arlington, TX). Prior to each reaching motion, the fingers to be used were dabbed into a black ink pad, such that the fingers left an approximately round, black mark (approximately $1 \mathrm{~cm}$ in diameter) where they had touched the paper.

Design and procedure. All participants completed five tasks with each of the four stimuli.

1. In the relative judgment task (four trials), participants were asked to complete the sentence, "If the horizontal line is 100 units long, the vertical is _ units long."

2. In the metric judgment task (eight trials), participants were asked to estimate the horizontal and vertical extent of all four figures in millimeters. (Horizontal and vertical responses for Tasks 2, 3, and 4 were conducted in separate trials.)

3. In the line-segmenting task (eight trials), participants were asked, as in Experiment 3, to demarcate a segment of a horizontal line such that it equaled the perceived length of the horizontal and vertical extents of all four displays.

4. In the two-fingered reach task (eight trials), participants were asked to make an open-loop reach for the horizontal and vertical extents of all four displays.

5. In the three-fingered reach task (four trials), participants were asked to reach for the two-dimensional stimuli depicted in Figures 9a and $9 \mathrm{~b}$ by using three fingers as pictured in Figures $9 \mathrm{c}$ and $9 \mathrm{~d}$. For the two-fingered and three-fingered reaching tasks, the hand was initially held out of view under the table surface. Only after the eyes were closed was the reaching action begun; the hand was returned to its occluded location before the eyes were reopened. In combination with the continued use of two-dimensional stimuli, the eye closing ensured that participants received no tactile or visual feedback about the accuracy of their actions.

The stimuli and instructions for each of these 32 trials were printed on individual sheets of paper $(15.0 \times 21.6 \mathrm{~cm})$. The papers containing the stimuli were thoroughly shuffled for each participant. The entire procedure was typically completed within about $30 \mathrm{~min}$. For the two-fingered reaches, the distance between the centers of the two marks was taken as the estimate of the target's size. For the three-fingered reaches, the distance between the center of the two finger marks was taken as the estimate of horizontal size; the distance between the center of the thumb mark and the line defined by the two finger marks was taken as the estimate of vertical distance (Figure 10).

\section{Results}

For each of the five tasks, the ratio of vertical to horizontal size estimates was calculated separately for the square and 
a

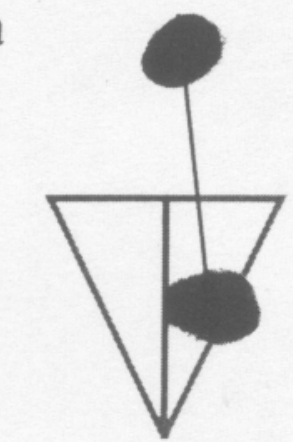

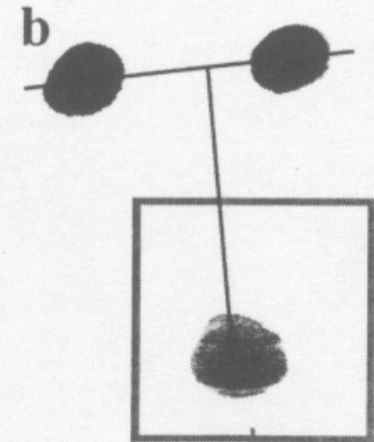

Figure 10. Typical data sheets from the two-fingered (Figure 10a) and three-fingered (Figure lob) reaching tasks. The fingers were coated with ink such that they left circular marks (examples shown here) on the paper where they had touched it. As in Experiment 1, two-fingered grip scaling was accurate even though the placement of the hand was often inaccurate. For three-fingered reaches, the vertical perception was assessed as the distance between the thumb and the line defined by the two fingers. Note that as in Experiment 1 , hand placement was much less inaccurate than grip scaling.

triangle stimuli for each participant. The effect of the shift from square to triangle stimuli was calculated in terms of the difference between these two ratios (Figure 11). A repeated measures ANOVA would have provided a convenient method of assessment for these data, but large disparities in task variance rendered this inappropriate. To provide a straightforward characterization of the performance in each of the tasks

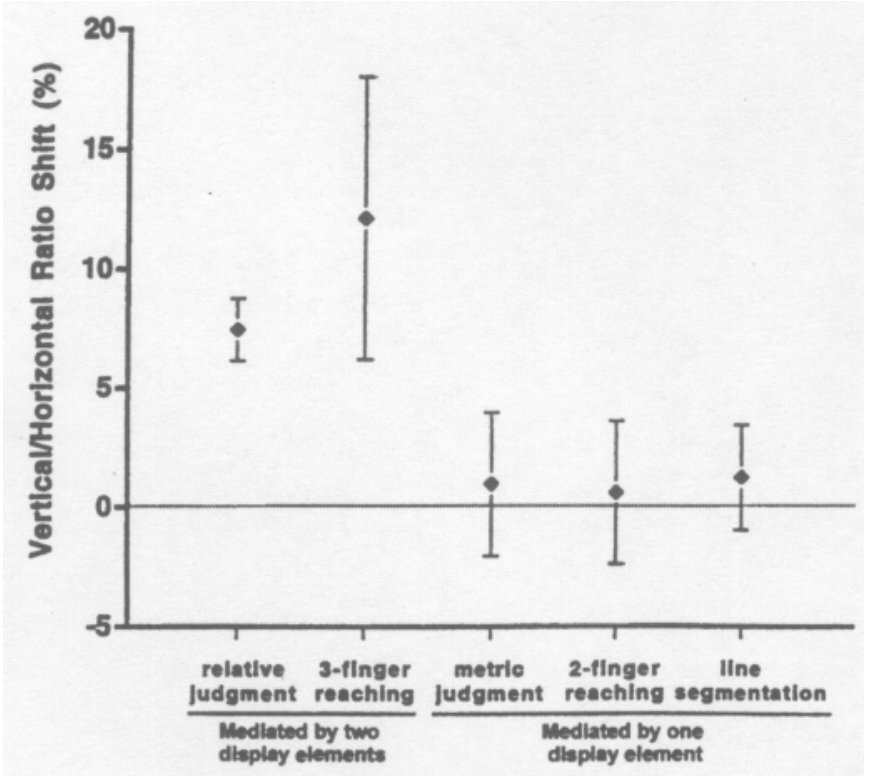

Figure 11. Percentage effect of the illusion on different size mediated tasks. Both multiple target tasks indicate a large effect of the horizontal-vertical illusion (relative judgment and threefingered reaching). All tasks that were mediated by a single element of a display were largely immune to the illusion (two-fingered reaching, metric judgment, and line segmentation). The gray line indicates the region of veridical performance; vertical error bars indicate $\pm 1 S E M$. and to allow for general comparisons, 95\% confidence intervals were calculated for the illusory effect for each task.

1. Relative judgment indicates that the triangle figures used in this study were subject to the horizontal-vertical illusion. The estimated ratios were significantly greater than $1: M=1.09 ; S E=0.01 ; t(22)=5.93, p<.001$. The mean estimate of relative height for the square was also greater than $1(M=1.01, S E=0.01)$, but this effect was not significant, $t(22)=0.99, p=.34$. The mean difference between these two implies that the switch from a square to triangle stimulus results in a mean $8 \%(S E=1.0)$ effect on relative judgment.

2. Metric judgments of length were highly variable, presumably because of the lack of feedback. Metric estimates ranged from 10 to $150 \mathrm{~mm}$ and averaged $134 \%$ ( $S E=5.8)$ of target size. When a ratio was calculated for each stimulus, for each participant, however, very consistent values emerged. Participants seemed not to have a good idea about how big a millimeter is, but their inaccurate conceptions were apparently consistent. The mean vertical/horizontal ratio for the triangle figures was 1.04 ( $S E$ $=0.02)$; for the square stimuli, the mean was $1.03(S E=$ 0.02 ). The overall effect of the triangle figure on the ratio of vertical to horizontal ratings was not significantly different from $0: M=0.01 ; S E=0.03 ; t(21)=0.32, p=.76$.

3 . The line-segmenting task produced results that were comparable to those of Experiment 3. For the square stimuli, vertical segments averaged $94 \%(S E=2.1)$ of target size; horizontal segments averaged $90 \%(S E=2.0)$ of target size. For the triangle stimuli, vertical segments averaged $89 \%(S E=2.3)$ of target size; horizontal segments averaged $85 \%(S E=2.0)$. The mean ratio of vertical to horizontal finger segments for the square stimuli was $1.05(S E=0.02)$; for the triangle, the mean ratio was $1.06(S E=0.02)$. The mean effect of this illusion on the ratio of vertical to horizontal estimates was $1 \%(S E=2.2)$. The effect was not significantly greater than $0, t(21)=0.56, p=.58$.

4. Two-fingered reaching was well scaled to the physical size of the target stimuli, as observed in Experiment 1. For the square stimuli, vertical finger gaps averaged 119\% (SE $=4.7$ ) of target size; horizontal finger gaps averaged $117 \%$ $(S E=4.7)$. For the triangle stimuli, vertical finger gaps averaged $112 \%(S E=4.8)$ of target size; horizontal finger gaps averaged $111 \%(S E=4.0)$. The mean ratio of vertical to horizontal finger gaps for the square stimuli was 1.00 ( $S E$ $=0.03)$; for the triangle, the mean ratio was $1.01(S E=$ 0.03 ). The mean effect of this illusion on the two-fingered reaching was $1 \%(S E=3.0)$. The effect is not significantly greater than $0, t(21)=0.20, p=.84$.

5. Three-fingered grip scaling was considerably less accurate than that observed in two-fingered reaching tasks, but both recorded distances were measurably affected by the size of the target element (Figure 12). For the square stimuli, the mean distance between the two fingers was $90 \%(S E=3.3)$ of target size; the mean distance between the thumb and fingers was $120 \%(S E=17.0)$ of target size. For the triangle stimuli, the mean distance between the two fingers was $83 \%(S E=2.9)$ of target size; the mean distance 


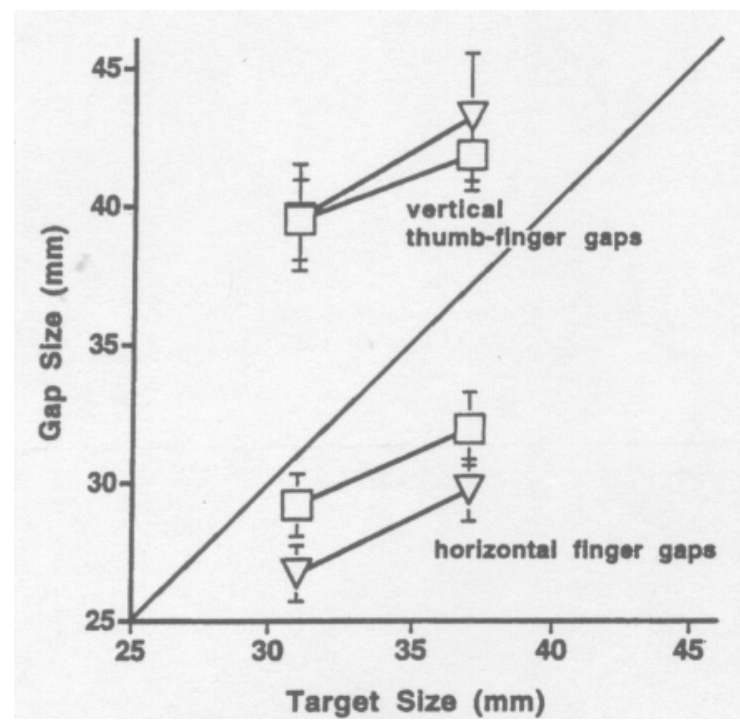

Figure 12. Grip size data for the square and triangle stimuli from the three-fingered reach task of Experiment 4. Three-fingered reaching, particularly the vertical component, was considerably less accurate than what has been previously observed in twofingered reaching tasks. However, both recorded distances were clearly a function of the size of the corresponding target element. The gray line indicates the region of veridical performance; vertical error bars indicate $\pm 1 S E M$.

between the thumb and fingers was $122 \%(S E=27.6)$ of target size.

The fact that the mean thumb-finger distance was larger than the finger-finger distance even for the square stimulus indicates that there is a biomechanical tendency in the direction of the horizontal-vertical illusion. As with the other tasks, however, what is of primary importance is whether an increase in the horizontal-vertical illusion was caused by a switch from the square stimulus to the triangle stimulus. The mean ratio for the square stimuli was 1.37 $(S E=0.29)$; for the triangle, the mean ratio was 1.49 ( $S E=$ $0.34)$. The switch from the square stimulus to the triangle stimulus resulted in a $12 \%$ illusory effect, and the effect of the illusion was marginally significant: $M=0.12 ; S E=$ $0.06 ; t(21)=2.05, p=.05$.

A closer look at the data reveals that the illusion had little effect on the thumb-finger distance, for which the mean shifted by only $2 \%$. The distance between the two fingers, however, was significantly smaller in the triangle condition, $t(21)=2.54, p=.02$, indicating a clear effect of the horizontal-vertical illusion on this component of grip scaling.

\section{Discussion}

1. The relative judgment task provides evidence that the triangle figure devised for this study, although different than the standard horizontal-vertical stimulus, does produce an analogous, somewhat smaller, illusory effect.

2. The metric judgment task replicates the main finding of Experiment 2 in a situation without feedback. The practice trials of Experiment 2 seem to have influenced the overall accuracy of estimates but not their near immunity to the effects of this illusion.

3. The line-segmenting task replicates the finding of Experiment 3 by using a new stimulus; again, these judgments made about single display elements exhibit a much smaller susceptibility to the illusion than relative judgment.

4. The two-fingered reaching task replicates the previous finding that grip scaling is largely unaffected by this pictorial illusion.

5. As predicted, the three-fingered task was greatly affected by the illusion; the presence of the bisecting vertical line resulted in significantly smaller horizontal grip sizes. As with a perceptual judgment task, when the motor action task is mediated by multiple elements of the display, the illusion exerts an effect.

\section{General Discussion}

Experiment 1 extended the findings of Aglioti et al. (1995), eliminating several classes of alternative explanations for the reported distinction between grip scaling and relative judgment behaviors and demonstrating the same trend with a different illusory display. Participants' performance on the metric judgment task of Experiment 2 and the segment-marking task of Experiment 3 was very similar to that observed for grip scaling and very different from relative judgment performance, suggesting that judgment behaviors that are based on a single element of the horizontal-vertical display are just as accurate as two-fingered grip scaling actions. Experiment 4 extends this account, providing evidence that when grip-scaling action is simultaneously mediated by the size of two different elements of a display, it is just as susceptible to the horizontal-vertical illusion as relative judgment.

In addition to the relative versus absolute distinction, there are indications that other variables are important for the control of size-mediated reaching. Post and Welch (1996) have reported that open-loop reaching is susceptible to the Mueller-Lyer illusion. This is not in conflict with the claims made here; we have only reported data from studies of the horizontal-vertical illusion. The prediction that does follow directly from our reasoning is that because this illusion affects two-fingered grip scaling, it should also affect absolute, metric judgment.

Additionally, Yang et al. (1998) have recently reported that absolute judgments of the size of long poles (2-20 feet) viewed from a distance of 45 feet are susceptible to the horizontal-vertical illusion. The length of the pole is greatly overestimated when oriented vertically, but not horizontally. It thus seems that if one uses stimuli that are placed at a much greater viewing distance, then different size and depth perception processing emerges. ${ }^{4}$ Our data imply that if some process is found to affect absolute judgment, then it should also affect actions that are mediated by that absolute information. On the basis of the results of Yang et al., we predict that open-loop, two-fingered grip scaling would be

\footnotetext{
${ }^{4}$ This specific prediction is described in great detail by Cutting and Vishton (1995).
} 
strongly influenced by the horizontal-vertical illusion if a participant were to view an inverted-T stimulus from a distance of several meters, then close his or her eyes, walk several steps forward, and reach for an element of the display. ${ }^{5}$

Overall, it seems that the striking distinction between grip scaling and overt judgment performance found in Experiment 1 is best described as a dissociation between relative and absolute size perception, rather than a dissociation between perception and action systems. How does this bear on the two-visual-systems hypothesis? The result runs counter to the suggestion by Aglioti et al. (1995) that the response behavior itself--skilled action versus perceptual judgment--is the crucial determiner of whether a pictorial illusion will exert a substantial effect on performance. It also runs counter to the more extensive interpretation of this work presented by Milner and Goodale (1995):

Although the perception of size is clearly affected by such contextual manipulations, it is possible that the processing responsible for the [Titchener circles] illusion occurs wholly within the ventral stream. If so, then the calibration of size dependent motor outputs, such as grip aperture during prehension, might not be affected. (p. 167)

Milner and Goodale went on to say that,

Mechanisms ... in which the relations between objects in the visual array play a crucial role in scene interpretation are clearly central to perception and on our model likely to be associated with the ventral stream. In contrast, the execution of a goal-directed act such as prehension depends on size computations that can be restricted to the target itself. (pp. 169-170)

Such claims suggest that judgment behaviors that rely on the representations present in the ventral stream should be susceptible to pictorial illusions, whereas action behaviors such as prehension should not. The four experiments presented here, although limited in scope, indicate that this is not necessarily the case.

However, the current results can be interpreted within the structure of the general two-visual-systems hypothesis. Provisions of the theory discuss ways in which the two streams must continually cooperate; both types of information are essential for the successful performance of any nontrivial task (Milner \& Goodale, 1995, pp. 88-92). Perhaps the participants in our study made use of the dorsal stream representations to accurately perform metric judgment and line segmentation tasks. Perhaps the ventral stream impinges on the dorsal stream's ordinary control of visually guided reaching when a three-fingered reach is initiated. These hypotheses could be tested using currently available brain imaging techniques that are sensitive to increased activation of the individual streams.

However, findings that indicate that the dorsal stream can be recruited for perceptual judgment would be consistent with the view that the dorsal stream, although extensively used for motor control tasks, is not specifically dedicated to those tasks but instead contains information that may be used for all tasks. An analogous statement could be made about a finding that the ventral stream contains information that is accessible for any and all types of behaviors. ${ }^{6}$
Further, provisions of the Milner and Goodale (1995) theory indicate that there are many different types of spatial representations present in both the ventral and the dorsal streams. It may be true that although the dorsal stream contains primarily egocentric representations that are largely immune to pictorial illusions, there are some representations that our particular methods have accessed that are not immune. Similarly, it may be true that the ventral stream contains some representations that are immune to pictorial illusions and some representations that are not.

A great deal of neuroanatomical, neurological, and electrophysiological evidence suggests the presence of two somewhat independent streams in the human visual cortex. We have presented only four studies of a single pictorial illusion. Any conclusions made on the basis of these experiments must be inherently preliminary in the face of such an edifice. What our results suggest, however, is that great caution must be exercised in translating this expansive theory into concrete predictions about the operating characteristics of normal, perceptually mediated action in humans.

\footnotetext{
${ }^{5}$ Haffenden and Goodale (1997) have reported that size perception for grip scaling is only maintained for $2 \mathrm{~s}$ after an object is removed from view. For such a study, the distance would need to be covered within this 2-s window.

${ }^{6}$ In fact, studies of pantomimed reaching that is based on remembered object size have already begun to explore this possibility (Goodale et al., 1994).
}

\section{References}

Aglioti, S., DeSouza, J. F. X., \& Goodale, M. A. (1995). Size contrast illusions deceive the eye but not the hand. Current Biology, 5, 679-685.

Avery, G. C., \& Day, R. H. (1969). Basis of the horizontal-vertical illusion. Journal of Experimental Psychology, 81, 376-380.

Brenner, E., \& Smeets, J. B. J. (1996a). Hitting moving targets: Co-operative control of "when" and "where." Human Movement Science, 15, 39-53.

Brenner, E., \& Smeets, J. B. (1996b). Size illusion influences how we lift but not how we grasp an object. Experimental Brain Research, $111,473-476$.

Bridgeman, B., Kirch, M., \& Sperling, A. (1981). Segregation of cognitive and motor aspects of visual function using induced motion. Perception \& Psychophysics, 29, 336-342.

Bridgeman, B., Lewis, S., Heit, G., \& Nagle, M. (1979). Relationship between cognitive and motor-oriented systems of visual position perception. Journal of Experimental Psychology: Human Perception and Performance, 5, 692-700.

Castiello, U., Bonfilioli, C., \& Bennett, K. M. B. (1996). How perceived object dimension influences prehension. NeuroReport, 7 , 825-829.

Castiello, U., \& Jeannerod, M. (1991). Measuring time to awareness. NeuroReport, 2, 797-800.

Castiello, U., Paulignan, Y., \& Jeannerod, M. (1991). Temporal dissociation of motor responses and subjective awareness: A study in normal subjects. Brain, 114, 2639-2655.

Cormack, E. O., \& Cormack, R. H. (1974). Stimulus configuration and line orientation in the horizontal-vertical illusion. Perception \& Psychophysics, 16, 208-212.

Cutting, J. E. (1986). Perception with an eye for motion. Cambridge, MA: MIT Press. 
Cutting, J. E., \& Vishton, P M. (1995). Perceiving layout: The integration, relative dominance, and contextual use of different information about depth. In W. Epstein \& S. Rogers (Eds.), Handbook of perception and cognition: Vol. 5. Perception of space and motion (pp. 69-117). New York: Academic Press.

Gentilucci, M., Daprati, E., Gangitano, M., Saetti, M. C., \& Toni, I. (1996). On orienting the hand to reach and grasp an object. NeuroReport, 7, 589-592.

Gentilucci, M., Daprati, E., Toni, I., Chieffi, S., \& Saetti, M. C. (1995). Unconscious updating of grasp motor programs. Experimental Brain Research, 105, 291-303.

Glaser, A. L., \& Slotnick, B. M. (1995). Visual inspection alone produces a decrement in the horizontal-vertical illusion. Perceptual \& Motor Skills, 81, 323-330.

Goldstein, E. B. (1996). Sensation \& perception (4th ed.). New York: Brooks Cole.

Goodale, M. A., Aglioti, S., \& DeSouza, J. F. X. (1994). Visual illusions affect perception but not prehension. Society for Neuroscience Abstracts, 20, 1666.

Goodale, M. A., Jakobson, L. S., \& Keillor, J. M. (1994). Differences in the visual control of pantomimed and natural grasping movements. Neuropsychologia, 32, 1159-1178.

Goodale, M. A., \& Milner, D. A. (1992). Separate visual pathways for perception and action. Trends in Neuroscience, 15, 20-25.

Goodale, M. A., Milner, D. A., Jakobson, L. S., \& Carey, D. P (1991, January 10). A neurological dissociation between perceiving objects and grasping them. Nature, 349, 154-156.

Goodale, M. A., Pelisson, D., \& Prablanc, C. (1986, April 24). Large adjustments in visually guided reaching to not depend on vision of the hand or perception of target displacement. Nature, 320, 748-750.

Haffenden, A. M., \& Goodale, M. A. (1997). Temporal properties of the mechanisms underlying visually guided prehension [Abstract 4568]. Investigative Ophthalmology \& Visual Science, 38, S988.

Higashiyama, A. (1992). Anisotropic perception of visual angle: Implication for the horizontal-vertical illusion, overconstancy of size, and the moon illusion. Perception \& Psychophysics, 51, 218-230.

Higashiyama, A. (1996). Horizontal and vertical distance perception:The discorded-orientation theory. Perception \& Psychophysics, 58, 259-270.

Howard, L. A., \& Tipper, S. P (1997). Hand deviations away from visual cues: Indirect evidence for inhibition. Experimental Brain Research, 113, 144-152.

Jakobson, L. S., \& Goodale, M. A. (1991). Factors affecting higher-order movement planning: A kinematic analysis of human prehension. Experimental Brain Research, 86, 199-208.

Jeannerod, M. (1984). The timing of natural prehension movements. Journal of Motor Behavior, 16, 235-254.

Jeannerod, M. (1988). The neural and behavioural organization of goal directed movements. Oxford, England: Oxford University Press.

Jeannerod, M. (1994). Object oriented action. Insights into the reach to grasp movement: Vol. 105. Advances in psychology (pp. 3-15). Amsterdam: North-Holland/Elsevier Science.

Jeannerod, M. (1997). The cognitive neuroscience of action. Oxford, England: Blackwell.
Jeannerod, M., Arbib, M. A., Rizzolatti, G., \& Sakata, H. (1995). Grasping objects: The cortical mechanisms of visuomotor transformation. Trends in Neurosciences, 18, 314-320.

Kubi, E., \& Slotnick, B. M. (1993). The horizontal-vertical illusion: Transfer of illusion decrement. Perceptual \& Motor Skills, 77, 339-347.

Loomis, J. M., Da Silva, J. A., Fujita, N., \& Fukusima, S. S. (1992). Visual space perception and visually directed action. Journal of Experimental Psychology: Human Perception and Performance, 18, 906-921.

Milner, A. D., \& Goodale, M. A. (1995). The visual brain in action. Oxford, England: Oxford University Press.

Post, R. B., \& Welch, R. B. (1996). Is there a dissociation of perceptual and motor responses to figural illusions? Perception, 25, 569-581.

Proffitt, D. R., Bhalla, M., Gossweiler, R., \& Midgett, J. (1995). Perceiving geographical slant. Psychonomic Bulletin \& Review, 2, 409-428.

Schiffman, H. R., \& Thompson, J. G. (1975). The role of figure orientation and apparent depth in the perception of the horizontalvertical illusion. Perception, 4, 79-83.

Servos, P., Goodale, M. A., \& Jakobson, L. S. (1992). The role of binocular vision in prehension: A kinematic analysis. Vision Research, 32, 1513-1521.

Smeets, J. B. J., \& Brenner, E. (1995). Perception and action are based on the same visual information: Distinction between position and velocity. Journal of Experimental Psychology: Human Perception and Performance, 21, 19-31.

Teghtsoonian, M. (1972). Apparent length as a function of tilt does not depend on orientation of the standard. Journal of Experimental Psychology, 94, 191-197.

Tipper, S. P., Howard, L. A., \& Jackson, S. R. (1997). Selective reaching to grasp: Evidence for distractor interference effects. Visual Cognition, 4, 1-38.

Todd, J. T., \& Norman, J. F. (1995). The visual discrimination of relative surface orientation. Perception, 24, 855-866.

Todd, J. T., \& Reichel, F. D. (1989). Ordinal structure in the visual perception and cognition of smoothly curved surfaces. Psychological Review, 96, 643-657.

Ungerleider, L. G., \& Mishkin, M. (1982). Two cortical visual systems. In D. J. Ingle, M. A. Goodale, \& R. J. W. Mansfeld (Eds.), Analysis of visual behavior (pp. 549-586). Cambridge, MA: MIT Press.

Verrillo, R. T., \& Irvin, G. (1979). Absolute estimation of line length as a function of orientation and contrast polarity. Sensory Processes, 3, 261-274.

Yang, T. L., Wade, M. M., \& Proffitt, D. R. (1998). Overestimation of heights is greater for objects in immersive compared to non-immersive displays. Retrieved September 5, 1998 from the World Wide Web: http://minerva.acc.Virginia.edu/-perlab/ heightover.dir/heightover.html.

Received November 24, 1997

Revision received September 8, 1998 Accepted November 2, 1998 